\title{
CityView: A Vehicle Based Image and Data Collection for Smart Cities
}

\author{
Ngurah Indra ER \\ IRISA/IMT-Atlantique, Rennes - France \\ \& \\ Udayana University, Bali - Indonesia \\ indra@unud.ac.id
}

\author{
Kamal Deep SINGH \\ Laboratoire Hubert Curien \\ Universite Jean Monnet \\ Saint-Etienne - France \\ kamal.singh@univ-st-etienne.fr
}

\author{
Jean-Marie BONNIN \\ IRISA / IMT-Atlantique - INRIA \\ Rennes - France \\ jean-marie.bonnin@irisa.fr
}

\begin{abstract}
The development of innovative applications for smart cities has been made possible by the rise of Internet of Things. The situational viewing and surveillance in cities is one such category of applications which can benefit from various networking solutions available to transport images or data from installed sensor cameras. In this paper, we propose and evaluate a citywide image and data collection service based on Vehicular Delay-Tolerant Networks (VDTN) and a simple hierarchical routing mechanism named Data Collection for Low Energy Devices (DC4LED). We study the networking performance in terms of increasing image sizes that can be transported with respect to varying vehicular density in city. We focus mainly on two technologies for sensors to vehicles communications: ZigBee and ITS-G5.
\end{abstract}

\section{INTRODUCTION}

In the current era, with the rise of Internet of Things (IoT), innovative applications for smart cities have been gaining momentum [1], [2]. One such area of novel applications is for a city's situational viewing and surveillance. Stationary image sensors or low cost cameras can be installed throughout the city to capture images for direct display or further analysis. Such a system can provide services such as detection of a crowded area, information for tourists, traffic jam detection, snow buildup detection, weather check and emergencies, among others. Note that the image quality required for each application may vary. For some types of services, even low-resolution images might be adequate, for example: differentiating between crowded and not so crowded area, distinguishing between traffic jam and regular traffic, weather check or to recognize the snow build-up on the road. On the other hand, high resolution images may be required when counting the number of people or vehicles, estimating the amount of snow build-up, or even: conducting face recognition.

From the networking point of view, we are interested in questions such as what are the networking solutions available for such applications? What is their capacity and performance in terms of QoS parameters? Up to what size of images or data can be transported using such solutions?

In this paper:

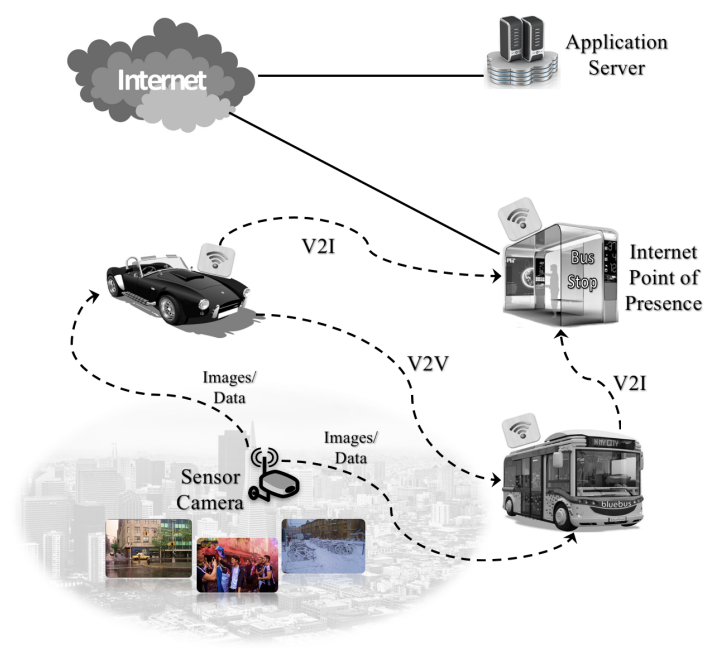

Fig. 1. The Vehicle-Based Image and Data Collection Architecture Overview

- We propose and evaluate a citywide image and data collection service for delay-tolerant applications. It is based on VDTN as shown in Figure 1.

- We study our VDTN solution's network performance in terms of delivery ratio, latency and data or image sizes that can be transported.

- We focus on studying two technologies that can be implemented to opportunistically connect sensor cameras with vehicles: ZigBee and ITS-G5 Vehicleto-infrastructure (V2I), and discuss ways to further increase its performance.

Some trade-offs and criteria should be taken into consideration before choosing the network solution to implement. The first criteria can be the data latency that can be tolerated by the application. Some applications need to receive data in real-time or near real-time, and some others can tolerate higher latency. The second is the bandwidth of the network available to transfer the corresponding resolution of images from the sensor camera. Here the solutions like the Long Range Wide Area Networking (LoRaWAN) will have a disadvantage that they only support low bandwidth not suitable for 
transport images. The third is the cost incurred by the network utilization, where networks such as cellular networks may cost more. Nevertheless, given the cost of integrating cellular communication capability in communicating objects and the cost of the communication, a few works explore the usage of LoRaWAN network to transport images.

We organize the rest of this paper as follows. Section II describes related works and how our work differs from them. Section III presents the vehicle-based image and data collections service in more details. Section IV describes the simulation setup. Section V presents and discusses simulation results. Lastly, some conclusions and the possibility of future works are provided in Section VI.

\section{RELATED WORKS}

Previous works in [3] and [4] have developed an image collection solution that utilized off-the-shelves components to gain flexibility and modularity. They implemented LoRa technology for low-power and longrange communications. Their solution, which requires a dedicated access network, can only transmit an image of size up to 1200 bytes every one hour, to be within the duty-cycle limitation set by ETSI. The limitations of LoRaWAN is further discussed in [5]. Therefore, this solution cannot be adopted for applications which need to transport larger images with higher frequency for viewing or image analysis. In our work, we propose a solution which can deliver images or data with larger sizes within minutes of their generation by the sensor camera.

Another IoT technology to consider is the IEEE 802.15.4 with ZigBee as its most widely deployed upper-layer enhancement. The implementation of IEEE 802.15.4 for smart metering is presented in [6], where they concluded that communications between devices ranging more than 25 meters can significantly harm the reliability. In [7], the authors presented their proof-ofconcept for the feasibility of ZigBee's data collection solution using a vehicle as a mobile sink. They also pointed out certain limitations for the ZigBee implementation in specific applications, which necessitates some improvements. Our work will push further the capability of the technology to deliver larger size of data or images.

The most recent technology designated for vehicular communications is the IEEE $802.11 \mathrm{p}$, with ITS-G5 as its standardization for Intelligent Transportation Systems (ITS) in Europe. It allows Vehicle-to-Vehicle (V2V) and Vehicle-to-Infrastructure (V2I) communications, which can seamlessly be extended for communications with objects in the environment, i.e. the Vehicle-to-Everything (V2X) communications. An experimental analysis of the technology presented in [8] concluded that $\mathrm{V} 2 \mathrm{~V}$ communications without noticeable decrease in bitrate can be achieved if the distance is not exceeding $300 \mathrm{~m}$ in
TABLE I

Specifications of The Communications Technologies

\begin{tabular}{|c|c|c|c|}
\hline \multirow{2}{*}{ Specifications } & \multicolumn{3}{|c|}{ Link Technologies } \\
\hline & $\begin{array}{l}\text { IEEE } \\
\text { 802.15.4 } \\
\text { (Zigbee) }\end{array}$ & $\begin{array}{l}\text { IEEE } \\
\text { 802.11p } \\
\text { (ITS G-5) }\end{array}$ & LoRaWAN \\
\hline Type of networks & Opportunistic & Opportunistic & Centralized \\
\hline $\begin{array}{l}\text { Communication } \\
\text { Range - Urban } \\
(\max )\end{array}$ & $100 \mathrm{~m}$ & $1 \mathrm{~km}$ & $5 \mathrm{~km}$ \\
\hline $\begin{array}{l}\text { Data Transfer Rate } \\
(\max )\end{array}$ & $250 \mathrm{kbps}$ & $6 \mathrm{Mbps}$ & $50 \mathrm{kbps}$ \\
\hline \multicolumn{4}{|c|}{ Size of a single image that can be forwarded } \\
\hline $1.2 \mathrm{~KB}$ & Yes & Yes & Yes \\
\hline $12 \mathrm{~KB}$ & Yes & Yes & No \\
\hline $120 \mathrm{~KB}$ & No & Yes & No \\
\hline $1.2 \mathrm{MB}$ & No & Yes & No \\
\hline
\end{tabular}

urban area. Furthermore, the technology have also been utilized in [9] for a successful V2V video transmission. It demonstrated that on an average of $4 \mathrm{Mbps}$ bandwidth is achievable for exchanging video during car overtaking scenario. In a scenario of data collection from stationary sensors, the contact duration with moving vehicles is generally very brief and the connection is intermittent. Thus, we employ VDTN solution which is known for its store, carry and forward approach [10].

Our work in [11] concluded that for small-sized sensor data, such as air quality measurements, a simple hierarchical routing mechanism for VDTN, which we named Data Collection for Low Energy Devices (DC4LED), could be utilized. We showed that it was a high performing data collection service for delay-tolerant applications in smart cities. However in [12], we also observed that disparity of vehicle's mobility affects data collection from sensors in some areas of the city. In some parts of the city, a large amount of data from sensors stays waiting very long before it gets picked by passing vehicles. Some data may even have to be dropped due to exceeding its time-to-live (TTL) or due to buffer overflow in sensors.

Image collection scenario is different. First the data size will be relatively higher. Such cameras may have bigger buffer capacity and also they will be able to choose from a broader range of networking technologies. Thus, we evaluate the performance of the VDTN routing strategy for the collection of bigger sized data coming from sensor cameras.

\section{Vehicle-Based Image and Data COLLECTION SERVICE}

The focus of our current work is to provide image or data collection service for delay-tolerant applications. This service can be provided by an opportunistic network such as VDTN via its V2X communication capabilities. The open standardization and the use of unlicensed spectrum have been the key driver for the implementation of the technology in both public and private sectors. We focus on two link layer technologies: ZigBee and 


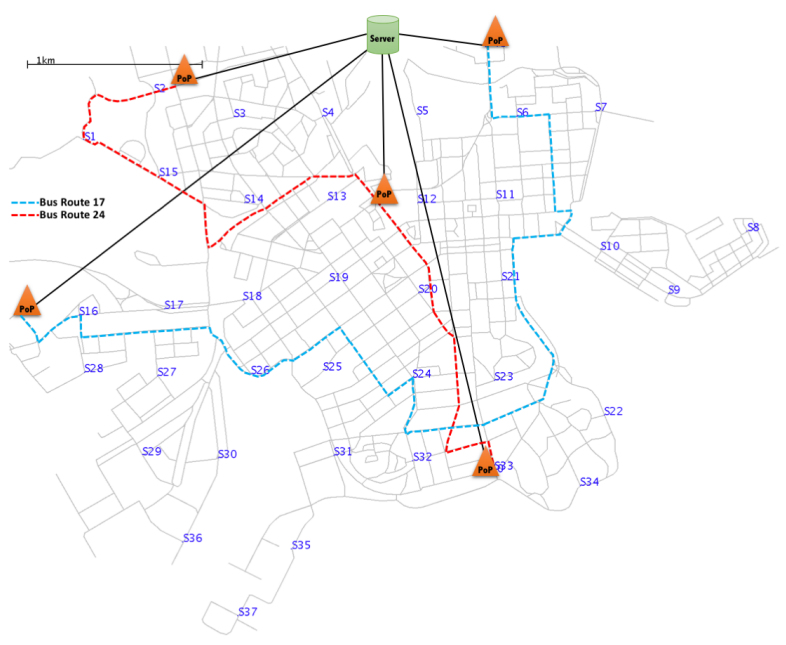

Fig. 2. Simulation Overview

ITS-G5 for opportunistic networking. Table I compares their specifications with parameter values corresponding to their respective standardization. It also shows specifications of LoRaWAN for comparison. We already discussed the bandwidth and duty-cycle limitation of LoRaWAN for large-size image and data collection in the previous section, which make it unsuitable for our purposes.

Table I also shows four types of image sizes which we later use in our evaluation. The smallest image size of $1.2 \mathrm{~KB}$ is the size of images that can be delivered using LoRa, as discussed in the previous section. The largest image size is calculated from a 640x480 32 bpp raw RGBA color image, which divided by 8 bits makes it $1200 \mathrm{~KB}$ or 1.2 MB in size (of course, with compression, higher resolution images can be assumed, but we still consider sizes up to $1.2 \mathrm{MB}$ in this study). By assuming 10:1 JPEG compression ratio for the raw image, we arrive at the size of $120 \mathrm{~KB}$, and compressing it further with the same ratio makes the image size to be $12 \mathrm{~KB}$. We then devise two sets of simulation scenarios based on the available bandwidth for the two technologies in focus. The goal is to study the size of images the technology can deliver in the context of VDTN.

In the context of VDTN, DC4LED is a forwarding algorithm which implements a simple hierarchical routing scheme. Algorithm 1 details DC4LED's forwarding algorithm. The idea is to statistically assign a level to the nodes in the city, instead of having complex routing decisions and metrics. This level is based on their reliability and capability to deliver the data to the server.

DC4LED considers mobility features of vehicles such as buses, taxis, and cars. Buses follow fixed routes and almost always on the move during their service hours, which makes them advantageous for VDTN routing in the case where the internet Point-of-Presence (PoP) is along their path. Cars and taxis, on the other hand, can roam streets which are not passed by buses and can gather data from sensors located close to any streets in the city. Furthermore, taxis roam the cities more as compared to cars, and generally, cars make only a few trips and have longer stationary times.

Thus, based on differences in the mobility pattern, we consider cars as the least reliable forwarder among vehicles, while buses are the most reliable in the algorithm. Note that a node does not forward the data to another node if that node's hierarchical level is inferior or equal to the current node. Thus, a bus or a taxi will not forward the data to a car, etc.

Initialize Nodes: Server.Level $=6$; PoP.Level $=$

5; Tram.Level $=4$; Bus.Level $=3$; Taxi.Level $=$

2; Car.Level $=1$; Sensor.Level $=0$;

Input: Connected NeighbourNode

while (CurrentNode.Level ! =0) do

| if CurrentNode.Level < NeighbourNode.Level then I forward message;

end

Algorithm 1: DC4LED Hierarchical Routing Forwarding Decision.

Now let us consider two scenarios for VDTN routing. In scenario 1, all sensor cameras equipped with ZigBee link transmit images with the size of $1.2 \mathrm{~KB}$ and 12 $\mathrm{KB}$ to in-range vehicles. For $\mathrm{V} 2 \mathrm{~V}$ communications, we assume that ITS-G5 is used. By means of V2V communication the data is then forwarded using the DC4LED's hierarchical forwarding algorithm. The data is forwarded until it reaches one of the Internet Pointof-Presence (PoP) and then onto the central server. We do not include image sizes of $120 \mathrm{~KB}$ and 1.2 MB for the simulation in scenario 1, because ZigBee's available bandwidth will be much smaller than that is required to forward this size of data.

In scenario 2, all sensor cameras are assumed to be equipped with the ITS-G5 V2I link. They transmit images with the size of $1.2 \mathrm{~KB}, 12 \mathrm{~KB}, 120 \mathrm{~KB}$ and 1.2 $\mathrm{MB}$ to vehicles that are in-range. The forwarding algorithm from vehicles to the central server is again assumed to be DC4LED as in scenario 1. In this case, we assume that sensor cameras in the city are connected to a power source and thus have sufficient energy. Also they have larger buffer size for storing high-resolution images.

\section{Simulation SETUP}

We evaluate the performance by using the Opportunistic Networking Environment (ONE) simulator [13][14]. An overview of our simulations is shown in figure 2 . It illustrates centralized situational monitoring in the city of Helsinki, where 37 wireless image capturing cameras 
are placed almost evenly in an area of about $9 \mathrm{~km}^{2}$. Each camera is positioned approximately at 500 meters of other cameras which gave one image data from 37 locations in the city every 5 minutes.

Cars, taxis, and buses equipped with V2X capabilities then opportunistically collect data from in-range sensors to be delivered to the first PoP they encounter, which in turn relays data to a central server. We implement the DC4LED routing algorithm for forwarding the data hierarchically. We assume two bus routes in the city, bus routes 17 and 24, where a PoP is strategically placed at each end of bus routes. The last PoP is positioned in the city center, where traffic usually converges. This in total makes 5 PoPs available to pick up data from cars, taxis, and buses.

Now lets see some parameters which are common to all scenarios. Table II provides the parameters and values used in the simulation. Each data packet has a Time to Live (TTL) of 5 hours. We assume each sensor camera has enough buffer size to store all data that it generates during the simulation, to make sure that we solely evaluate the network performances. Each car and taxi with pseudo-random way-points and shortest-path map-based movement has two wireless interfaces. Buses with predefined way-points and routes also have two wireless interfaces identical to cars and taxis in each scenario, but they have different speed range: $10 \mathrm{~km} / \mathrm{h}$ to $50 \mathrm{~km} / \mathrm{h}$ for cars and taxis, and $10 \mathrm{~km} / \mathrm{h}$ to $30 \mathrm{~km} / \mathrm{h}$ for buses. The stationary time after arriving at each waypoint (or bus stop in the case of buses) is 1 to 120 minutes for cars, 1 to 5 minutes for taxis, and 5 to 30 seconds for buses. We also provide enough buffer size for each type of vehicle to store all data generated by all sensor cameras during the simulation. Each PoP has two interfaces; the first is an ITS-G5 V2I radio interface to connect to cars, taxis, and buses, and the second is a long-range IEEE 802.11 on $5 \mathrm{GHz}$ band, point to point interface $(5 \mathrm{~km}$ in range and data rate of $300 \mathrm{Mbps}$ ) for the link to the server. Such specifications are found in some commercial products. ${ }^{1}$ The server also has the same long-distance IEEE 802.11 radio connection $(5 \mathrm{~km}$ in range and data rate of $300 \mathrm{Mbps}$ ) to receive data from each PoP. We then assess the performance with an increasing number of cars and taxis with similar proportion, from 1 car and 1 taxi, which corresponds to vehicles density of 0.22 per $\mathrm{km}^{2}$, to 45 cars and 45 taxis, which corresponds to vehicles density of 10 per $\mathrm{km}^{2}$. We run each scenario ten times with different initial positioning of cars and taxis in the city, and accumulate all results to come up with convincing trends.

\footnotetext{
${ }^{1}$ https://greentech-electronics.com/product/ens500/.

TABLE II

SIMULATION PARAMETERS AND VALUES

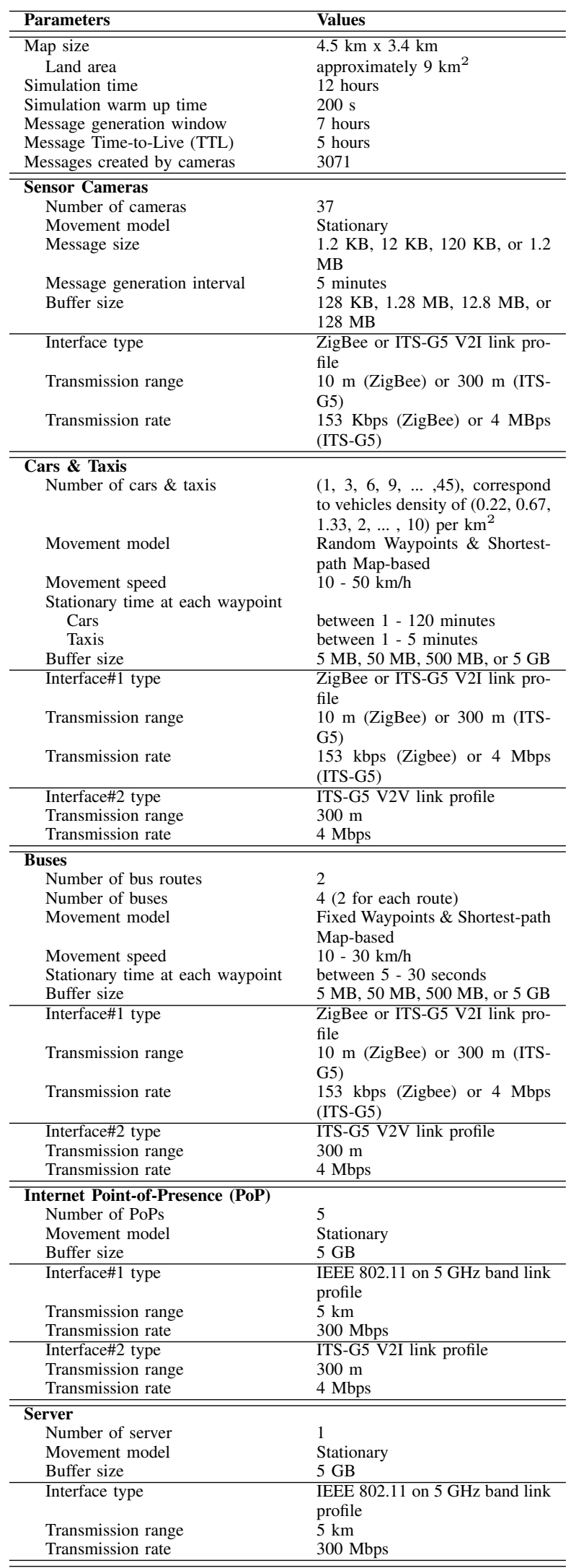




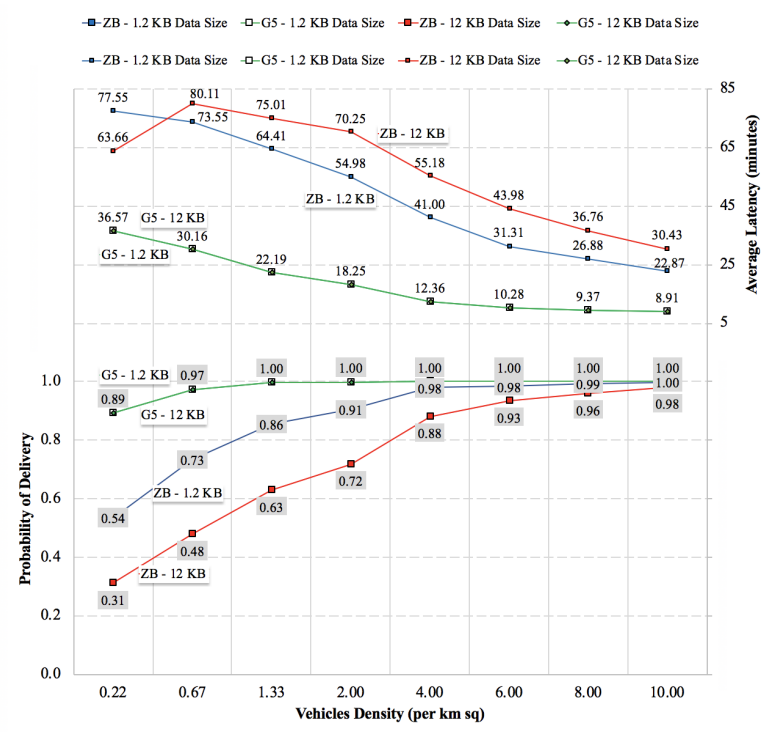

Fig. 3. Comparison of Delivery Probability and Average Latency for $1.2 \mathrm{~KB}$ and $12 \mathrm{~KB}$ Data Size

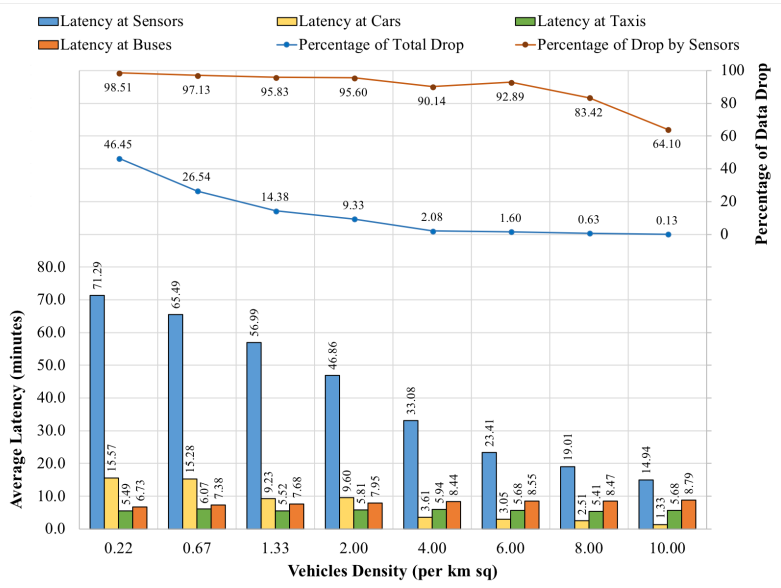

Fig. 4. Percentage of Data Drop and Distribution of Latency (ZigBee Sensors to Vehicles Link)

\section{RESULTS AND DISCUSSION}

Results presented in this section show the overall performance of the VDTN based image and data collection. The focus is on two technologies implemented in sensor cameras to connect to the vehicular networks. Remember that our research question is to find out: up to what sizes of image and data can be transported using VDTN with different link technologies?

Figure 3 shows the delivery probability and the average latency for the collection of $1.2 \mathrm{~KB}$ and $12 \mathrm{~KB}$ size images with increasing vehicular density. The figure also emphasizes the performance difference between ZigBee and ITS-G5 V2I implementation in the sensor camera. Generally, the part showing the probability of delivery illustrates an increasing trend with more vehicles involved in the data collection.
In scenario 1, where sensor cameras use ZigBee to connect to vehicles, comparison between $1.2 \mathrm{~KB}$ and $12 \mathrm{~KB}$ image collection shows lower probability of delivery for the larger data size. This is due to the longer contact duration needed between sensors and vehicles to successfully forward larger data, i.e., the probability of successful data transfer in a short duration of contact will be higher for smaller data size. The contact duration is directly related to the vehicle's speed, where higher speed means shorter contact duration. The figure also shows that with higher vehicular density, where the number of slower moving vehicles is also statistically higher, a larger data size can be delivered more successfully.

In term of the average latency, Figure 3 shows the trends of decreasing latency with the increasing vehicles density. Comparison between $1.2 \mathrm{~KB}$ and $12 \mathrm{~KB}$ data sizes also emphasizes that forwarding larger data introduces higher latency.

In scenario 2, where sensor cameras use ITS-G5 V2I to connect to vehicles, comparison between $1.2 \mathrm{~KB}$ and $12 \mathrm{~KB}$ size image collection shows minor difference in the probability of delivery and average latency. The performance is almost the same due to the much larger bandwidth available for forwarding the image compared to the size of the image itself.

Lastly in figure 3, we can also observe the difference in performance caused by ZigBee and ITS-G5 V2I utilization in the sensor cameras. The figure shows that ITS-G5 connection (scenario 2), with its longer communications range and higher bandwidth, provides higher probability of delivery and lower average latency than ZigBee. The difference in performance is more apparent in a low vehicles density, for example: in the collection of $1.2 \mathrm{~KB}$ images and vehicles density of 0.22 per $\mathrm{km}^{2}$, the ITS-G5 probability of delivery is already as high as 0.89 , while for ZigBee it is only 0.54 . The probability is even worse for ZigBee for the collection of $12 \mathrm{~KB}$ images, where the value is as low as 0.31 . The average latency for ITS-G5 implementation is shown to be below 10 minutes during high vehicular density, which might be suitable for some delay-tolerant applications requiring lower latency.

Figure 4 emphasizes the percentages of data drop and the latency distribution for scenario 1, where the sensor cameras are connected via ZigBee, for the smallest image size of $1.2 \mathrm{~KB}$. The line graphs which represent percentages of data drop, show two statistics. The line graph on top illustrates the percentages of data being dropped out of all 3071 data instances generated during the simulation. It shows a high drop percentages of $46.45 \%$ during the lowest vehicular density. The drop percentage decreases as the vehicle density increases. The drop percentages are as low as $0.13 \%$ at the highest vehicle density simulated. Furthermore, the line graph 


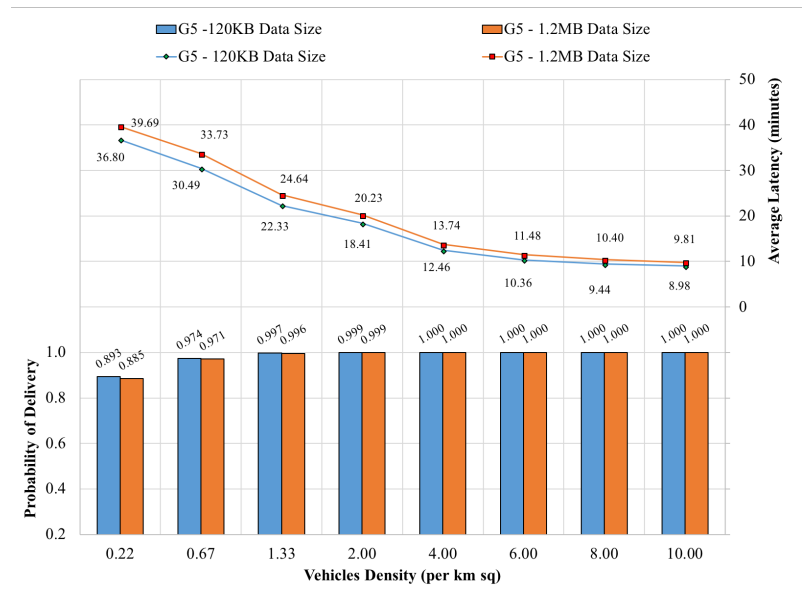

Fig. 5. Delivery Probability and Average Latency for $120 \mathrm{~KB}$ and 1.2 MB Data Size

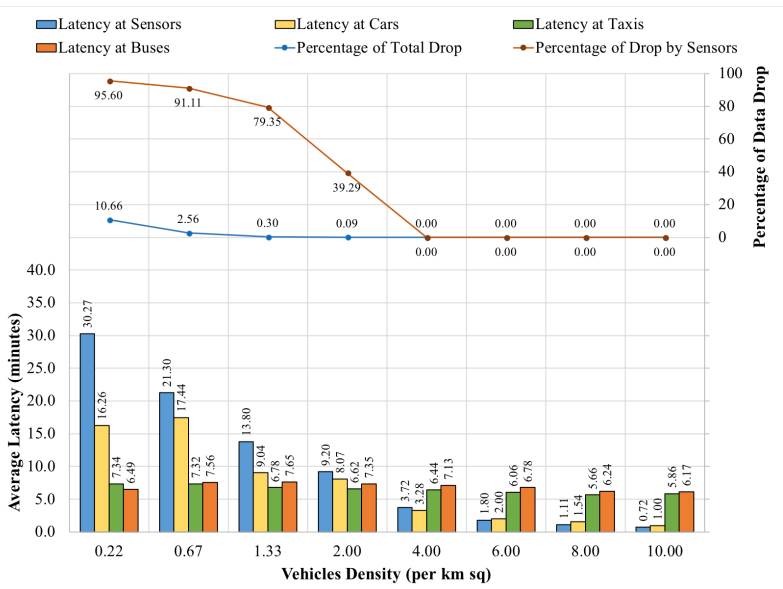

Fig. 6. Percentage of Data Drop and Distribution of Latency (ITS-G5 Sensors to Vehicles Link)

in the middle shows the percentages of data being dropped by sensors, out of all dropped data. The value is very high, $98.51 \%$ at the lowest vehicular density, and continues to be higher than $90 \%$ up to a vehicular density of 6 per $\mathrm{km}^{2}$, even though the overall data drop decreases significantly. This is mainly due to the short communication range offered by the technology, which limits the number of contacts with vehicles and also shortens the contact duration.

The bar graph in Figure 4 shows the distribution of average latency for the successfully delivered data from sensors to the central server in scenario 1. It shows significant data latency in sensors as compared to latency while being carried in vehicles. At the lowest vehicular density, data need to wait 71.29 minutes on an average in the sensor camera's buffer, before any vehicle is in the range to pick-up data. The latency decreases as more vehicles become available to collect data, but the latency at sensor camera's buffer continues to be higher than the latency in vehicles.

Figure 5 shows the delivery probability and the average latency of scenario 2. In scenario 2, sensor cameras use ITS-G5 V2I to connect to vehicles, for the collection of $120 \mathrm{~KB}$ and 1.2 MB images with increasing vehicular density. It shows a higher delivery probability and lower latency as compared to ZigBee's performance shown before. This is thanks to the ITS-G5's longer communication range between sensors and vehicles. At the lowest vehicular density of 0.22 per $\mathrm{km}^{2}$, the probability of delivery is already at 0.89 with an average latency slightly below 40 minutes. A comparison between 120 $\mathrm{KB}$ and 1.2 MB images transmission shows only slight differences in performance, i.e., of around 3 minutes lower average latency at the lowest vehicular density. As more vehicles are involved in the image collection, the probability of delivery becomes higher and the average latency gets lower, reaching slightly below 10 minutes at a vehicular density of 10 per $\mathrm{km}^{2}$.

Figure 6 emphasizes the percentages of data drop and the latency distribution for the second scenario with the image size of $120 \mathrm{~KB}$. It reveals more on the dynamics of the image collections process. On the percentages of data drop, we can observe a low value of $10.66 \%$ as compared to $46.45 \%$ for the ZigBee's implementation shown in Figure 4 at the same vehicular density of 0.22 per $\mathrm{km}^{2}$. The percentage of data drop by sensors is still dominant at a lower vehicular density, which continues to decrease as the density increases. The same trend can be seen for average latency, where predominantly the latency occurs in sensors before the vehicular density reaches 4 per $\mathrm{km}^{2}$. At a vehicular density of 4 per $\mathrm{km}^{2}$, when perfect delivery is reached, the average latency trend is reversed. At that point, the latency that occurs in sensors become lower than the latency in vehicles, particularly in taxis and buses. This shows that, given sufficient opportunities, data from sensors can be forwarded to vehicles faster than the data being forwarded from taxis and buses to PoPs. This points out that further latency reduction is possible by adding more PoPs in the city, naturally along the bus routes. The fact that only 2 bus routes with 4 buses are included in the simulation also opens the possibility to further reduce the latency by involving more buses and routes to the data collection process.

By reviewing all results, we can observe the dynamics due to different data sizes from sensors, connection bandwidth between sensors and vehicles, and their communication ranges. A combination of larger data size, lower connection bandwidth, and shorter communication range leads to lower performances, while smaller data size, higher connection bandwidth, and a higher range of communication produces higher performances. This is important for planning the networking part of the data collection, to achieve the desired performance of 
the planned application. Furthermore, as communication technologies embedded in objects are usually deployed specifically for a given set of usages/applications, their networking capabilities are more or less already fixed.

Finally note that, in future it is highly likely that the vehicles will embark multiple technologies in order to cope with heterogeneous objects and needs. Thus, some technologies can be used in an adaptive way according to their performance in given situations. Another example is that some technologies like LoRaWAN can be used as a fallback solution. When in remote places the images are not being forwarded due to low vehicular density, then either just the meta-data or very low resolution may be forwarded using LoRaWAN. Such minimal data might still be useful depending on the nature of the application.

\section{CONCLUSIONS AND FUTURE WORKS}

In this paper, we presented and analyzed the performance of our VDTN based image or data collection with two possible technologies to link sensor cameras with vehicles. The VDTN routing using technologies such as ZigBee and ITS-G5, can forward low-resolution images or data with high delivery probability, provided enough vehicles are involved in the collection process. Highresolution images, on the other hand, can only be forwarded by ITS-G5 with its higher bandwidth and longer communications range. These characteristics proved to be significant in maximizing the delivery probability and minimizing the average latency, thus broadening the possibility of services that can be supported by VDTN. Furthermore, the trend in network performances are consistent with findings in our previous work: a simple routing scheme provides a low network overhead. This in turn is advantageous for its implementation scalability.

Indeed, some solutions need to be devised to raise performance in the case where some areas of the city might experience low vehicular density and mobility. Our future works will study possible strategies to address such issues, such as providing a mechanism for the sensor camera to forward critically-delayed images with its lower resolution through alternative means of communications with the server.

Finally, note that there can be security and privacy concerns with image capture depending on the type of application. They are beyond the scope of this paper. Nevertheless, in the case of public applications, one way to deal could be to blur the images of people and other sensitive information as is done in some street-view, map applications. Moreover, in our networkbased approach, end-to-end security is enforced through encryption directly between the object and the server.

\section{ACKNOWLEDGMENT}

This work is supported by a Doctoral Scholarship from the Indonesian Endowment Fund for Education (LPDP) of the Indonesian Ministry of Finance, under Contract Number PRJ-43/LPDP.3/2016.

\section{REFERENCES}

[1] A. Zanella, N. Bui, A. Castellani, L. Vangelista, and M. Zorzi, "Internet of Things for Smart Cities," IEEE Internet of Things Journal, vol. 1, no. 1, pp. 22-32, Feb. 2014.

[2] P. Rawat, K. D. Singh, H. Chaouchi, and J. M. Bonnin, "Wireless sensor networks: a survey on recent developments and potential synergies," The Journal of supercomputing, vol. 68, no. 1, pp. $1-48,2014$.

[3] C. Pham, "Low-cost, Low-power and Long-range Image Sensor for Visual Surveillance," in Proceedings of the 2nd Workshop on Experiences in the Design and Implementation of Smart Objects, ser. SmartObjects '16. New York, NY, USA: ACM, 2016, pp. 35-40, event-place: New York City, New York.

[4] - "Robust CSMA for long-range LoRa transmissions with image sensing devices," in 2018 Wireless Days (WD), Apr. 2018, pp. 116-122.

[5] F. Adelantado, X. Vilajosana, P. Tuset-Peiro, B. Martinez, J. Melia-Segui, and T. Watteyne, "Understanding the Limits of LoRaWAN," IEEE Communications Magazine, vol. 55, no. 9, pp. 34-40, Sep. 2017.

[6] A. F. d. S. Veloso, A. A. Rodrigues, J. V. V. Sobral, J. J. P. C. Rodrigues, M. S. S. Feitosa, and R. A. L. Rabelo, "An IoT Smart Metering Solution Based on IEEE 802.15.4," in 2018 IEEE Global Communications Conference (GLOBECOM), Dec. 2018, pp. 1-6.

[7] T. d. A. Oliveira and E. P. Godoy, "ZigBee Wireless Dynamic Sensor Networks: Feasibility Analysis and Implementation Guide," IEEE Sensors Journal, vol. 16, no. 11, pp. 4614-4621, Jun. 2016.

[8] F. A. Teixeira, V. F. e Silva, J. L. Leoni, D. F. Macedo, and J. M. S. Nogueira, "Vehicular networks using the IEEE 802.11p standard: An experimental analysis," Vehicular Communications, vol. 1, no. 2, pp. 91-96, Apr. 2014.

[9] J. Pereira, M. Diaz-Cacho, S. Sargento, A. Zuquete, L. Guardalben, and M. Luis, "Vehicle-to-Vehicle Real-Time Video Transmission through IEEE 802.11p for Assisted-Driving," in 2018 IEEE 87th Vehicular Technology Conference (VTC Spring), Jun. 2018, pp. 1-6.

[10] N. Benamar, K. D. Singh, M. Benamar, D. El Ouadghiri, and J.-M. Bonnin, "Routing protocols in vehicular delay tolerant networks: A comprehensive survey," Computer Communications, vol. 48, pp. 141-158, 2014

[11] N. I. Er, K. D. Singh, and J.-M. Bonnin, "DC4LED: A Hierarchical VDTN Routing for Data Collection in Smart Cities," in 2019 16th IEEE Annual Consumer Communications Networking Conference (CCNC), Jan. 2019, pp. 1-4.

[12] _ , "Contact Strategy for VDTN Data Collection in Smart Cities," in ICSGTEIS 2018 - International Conference on SmartGreen Technology in Electrical and Information Systems, Oct. 2018.

[13] A. Keranen, J. Ott, and T. Karkkainen, "The ONE Simulator for DTN Protocol Evaluation," in Proceedings of the 2nd International Conference on Simulation Tools and Techniques, 2009, pp. $55: 1-55: 10$.

[14] J. Dede, A. Forster, E. Hernandez-Orallo, J. Herrera-Tapia, K. Kuladinithi, V. Kuppusamy, P. Manzoni, A. b. Muslim, A. Udugama, and Z. Vatandas, "Simulating Opportunistic Networks: Survey and Future Directions," IEEE Communications Surveys Tutorials, vol. 20, no. 2, pp. 1547-1573, 2018. 